\title{
Effects of supplementation with antifreeze proteins on the follicular integrity of vitrified-warmed mouse ovaries: Comparison of two types of antifreeze proteins alone and in combination
}

\author{
Min Kyung Kim ${ }^{1,2}$, Hyun Sun Kong ${ }^{2,3}$, Hye Won Youm², Byung Chul Jee ${ }^{2,3}$ \\ 'Division of Biotechnology, College of Life Sciences and Biotechnology, Korea University, Seoul; ${ }^{2}$ Department of Obstetrics and Gynecology, Seoul National \\ University Bundang Hospital, Seongnam; ${ }^{3}$ Department of Obstetrics and Gynecology, Seoul National University College of Medicine, Seoul, Korea
}

Objective: The aim of this study was to analyze the effect of supplementing vitrification and warming solutions with two types of antifreeze proteins (AFPs) and the combination thereof on the follicular integrity of vitrified-warmed mouse ovaries.

Methods: Ovaries $(n=154)$ were obtained from 5-week-old BDF1 female mice $(n=77)$ and vitrified using ethylene glycol and dimethyl sulfoxide with the supplementation of $10 \mathrm{mg} / \mathrm{mL}$ of Flavobacterium frigoris ice-binding protein (FflBP), $10 \mathrm{mg} / \mathrm{mL}$ of type III AFP, or the combination thereof. Ovarian sections were examined by light microscopy after hematoxylin and eosin staining, and follicular intactness was assessed as a whole and according to the type of follicle. Apoptosis within the follicles as a whole was detected by a terminal deoxynucleotidyl transferase deoxyuridine triphosphate nick-end labeling assay.

Results: The proportion of overall intact follicles was significantly higher in the type III AFP-supplemented group (60.5\%) and the combination group (62.9\%) than in the non-supplemented controls $(43.8 \%, p<0.05$ for each). The proportion of intact primordial follicles was significantly higher in the FflBP-supplemented (90.0\%), type III AFP-supplemented (92.3\%), and combination (89.7\%) groups than in the non-supplemented control group ( $46.2 \%, p<0.05$ for each). The proportions of non-apoptotic follicles were similar across the four groups.

Conclusion: Supplementation of the vitrification and warming solutions with FfIBP, type III AFP, or the combination thereof was equally beneficial for the preservation of primordial follicles in vitrified mouse ovaries.

Keywords: Antifreeze protein; Fertility preservation; Ovary; Vitrification

\section{Introduction}

Radiotherapy and/or chemotherapy can be detrimental to the reproductive function of women, and can cause decreased ovarian function or ovarian failure [1]. Fertility preservation techniques, such as oocyte, embryo, or ovarian tissue cryopreservation, have come to

Received: Sep 13, 2016 · Revised: Dec 13, 2016 · Accepted: Jan 9, 2017 Corresponding author: Byung Chul Jee

Department of Obstetrics and Gynecology, Seoul National University Bundang Hospital, 82 Gumi-ro 173beon-gil, Bundang-gu, Seongnam 13620, Korea Tel:+82-31-787-7254 Fax:+82-31-787-4054 E-mail: blasto@snubh.org

This is an Open Access article distributed under the terms of the Creative Commons Attribution Non-Commercial License (http://creativecommons.org/licenses/by-nc/4.0/) which permits unrestricted non-commercial use, distribution, and reproduction in any medium, provided the original work is properly cited. play an important role in preserving the reproductive function of cancer survivors [2]. Ovarian tissue cryopreservation can be applied to cancer patients who require immediate cancer treatment and who have no spouse or partner, as well as to pre-pubertal girls [3-5].

Although ovarian tissue cryopreservation is a promising technique, some issues remain to be addressed before it can be considered to be securely established. Several cryoinjury-related events such as follicle loss, stromal cell destruction, and apoptosis can occur during the cryopreservation process [6]. In order to alleviate the cryoinjury of ovarian tissue and improve its survival, techniques are being developed to optimize cryoprotectants by modulating their composition and concentration, as well as through supplementation with various additives [7-9]. 
Antifreeze proteins (AFPs) are groups of polypeptides produced by vertebrates, insects, fungi, bacteria, and plants, and play a role in survival in below-zero temperatures [10]. DeVries and Wohlschlag [11] first discovered AFPs in the serum of Antarctic fish in 1969, and thereafter multiple animal studies have reported that AFPs had a positive effect on the survival of oocytes, embryos, and ovaries during cryopreservation $[12,13]$. AFPs have been reported to bind to ice crystals and inhibit their growth and recrystallization by lowering the freezing point below the equilibrium melting point [14]. AFPs also protect cell membranes from physical injury $[15,16]$. Bagis et al. [17] reported that the litter sizes of mice with freshly transplanted ovaries were comparable to those of mice with AFP-transplanted transgenic ovaries, whereas those from nontransgenic-transplanted ovaries were significantly smaller, which was argued to demonstrate the beneficial effects of AFPs on ovarian tissue vitrification. Therefore, it can be expected to reduce cryoinjury during cryopreservation and to enhance survival by maintaining the follicular structure in the mouse ovary.

Several previous studies have demonstrated that AFPs, including Flavobacterium frigoris ice-binding protein (FfIBP) and type III AFP, had a beneficial effect on the preservation of mouse oocytes or ovaries during vitrification and warming $[14,18]$. Lee et al. [13] showed that FfIBP and type III AFP, as well as glycosylated ice-binding protein, improved the quality of oocytes and embryos after the vitrification and warming of mouse oocytes. In addition, Lee et al. [19] demonstrated that supplementation with those AFPs had protective effects on follicle preservation during mouse ovarian tissue vitrification and warming.

In the present study, we investigated whether the combination of FfIBP and type III AFP improved the follicular integrity of vitrifiedwarmed mouse ovaries.

\section{Methods}

\section{Experimental animals and collection of mouse ovaries}

Seventy-seven 5-week-old female BDF1 mice (Orient Bio, Seongnam, Korea) were used. The mice were housed under a 12-hour light and 12 -hour dark cycle at $22^{\circ} \mathrm{C}$, and fed ad libitum in accordance with the guidelines established by the Institutional Animal Care and Use Committee of Bundang Hospital, Seoul National University. After 1 week of adaptation, the mice were sacrificed by cervical dislocation and their ovaries were resected.

\section{Vitrification and warming of mouse ovaries}

The mouse ovaries $(n=154)$ were randomly assigned into a nonsupplemented vitrified control group and three vitrified groups where the vitrification and warming solutions were supplemented with $10 \mathrm{mg} / \mathrm{mL}$ of FfIBP, $10 \mathrm{mg} / \mathrm{mL}$ of type III AFP, and the combina-
Table 1. Chemical properties of the two AFPs used in this study

\begin{tabular}{|c|c|c|}
\hline Variable & FflBP & Type III AFP \\
\hline Organism & $\begin{array}{l}\text { Flavobacterium } \\
\text { frigoris PS1 }\end{array}$ & $\begin{array}{c}\text { Ocean pout, wolffish, } \\
\text { eelpout }\end{array}$ \\
\hline Structure & $\beta$-Helix & Globular \\
\hline Size (kDa) & $\sim 25.3$ & 6.5 \\
\hline Thermal hysteresis valuea) & $2.5^{\circ} \mathrm{C}$ at $50 \mu \mathrm{M}$ & $\sim 1.5^{\circ} \mathrm{C}$ at $3 \mathrm{mM}$ \\
\hline
\end{tabular}

AFP, antifreeze protein; FfBP, Flavobacterium frigoris ice-binding protein.

a)The difference between freezing point and equilibrium melting point.

tion thereof. Each AFP concentration was determined as previously reported [13]. The chemical properties of the two AFPs used in this study are summarized in Table 1.

Vitrification was performed using a two-step method. Dulbecco's phosphate-buffered saline (DPBS; Gibco, Carlsbad, CA, USA) supplemented with 20\% fetal bovine serum (FBS, Gibco) was used as a basic medium. For the first step, the ovaries were equilibrated in a solution composed of 7.5\% ethylene glycol (EG; Sigma-Aldrich, St. Louis, MO, USA) and 7.5\% dimethyl sulfoxide (DMSO, Sigma-Aldrich) for 10 minutes. Then, the ovaries were transferred to a vitrification solution composed of 20\% EG, 20\% DMSO, and 0.5 M sucrose (Sigma-Aldrich) for 5 minutes. The ovaries were placed on an electron microscopic copper grid (JEOL, Tokyo, Japan) and immediately transferred to 1.5$\mathrm{mL}$ cryovials (Nunc, Roskilde, Denmark) that were already filled with liquid nitrogen. All procedures were performed at room temperature. For the warming procedure, the cryovials containing the vitrified ovaries were put into a $37^{\circ} \mathrm{C}$ water bath and then transferred to warming solutions serially supplemented with $1.0 \mathrm{M}, 0.5 \mathrm{M}, 0.25 \mathrm{M}$, and $0 \mathrm{M}$ sucrose for 5 minutes each. After warming, the ovaries were incubated in a-minimal essential medium (Welgene, Daegu, Korea) supplemented with $10 \% \mathrm{FBS}$ and $10 \mathrm{mlU} / \mathrm{mL}$ of recombinant follicle stimulating hormone (Serono, Geneva, Switzerland) for 30 minutes in a humidified incubator maintained at $37^{\circ} \mathrm{C}$ and $5.5 \% \mathrm{CO}_{2}$. This short incubation period was found to have enhanced the survival of mouse ovarian tissue in our previous studies $[7,18]$. The ovaries were washed and transferred to $1 \mathrm{~mL}$ of culture medium in an organ dish (BD Biosciences, San Jose, CA, USA) covered with oil.

\section{Histological analysis of ovarian follicles}

All the ovaries ( $n=154)$ were fixed with $4 \%$ paraformaldehyde, embedded in a paraffin block and serially sectioned at a thickness of 4 $\mu \mathrm{m}$. Only one section was obtained from each ovary. Some of the paraffin section slides $(n=77)$ were stained with hematoxylin and eosin (Merck, Darmstadt, Germany) and observed under light microscopy (Nikon, Tokyo, Japan) at $\times 400$ magnification. The follicles were assessed and counted if they included an oocyte. The follicle stage was assessed as primordial, primary, secondary, or antral ac- 
cording to previous guidelines [20]. As shown in Figure 1, the morphological integrity of each follicle was evaluated using the following criteria, with grade 1 considered the most intact form, as previously reported [21].

\section{1) Primordial/primary follicle}

Grade 1, spherical with even distribution of the granulosa cells; grade 2 , granulosa cells pulled away from the edge of the follicle but with the oocytes still spherical; grade 3, pyknotic nuclei, misshapen oocytes, or vacuolation.

\section{2) Secondary/antral follicle}

Grade 1, intact spherical follicle with evenly distributed granulosa and theca cells, a small space between granulosa and theca cells, and spherical oocytes; grade 2, intact theca cells, disrupted granulosa cells, and spherical oocytes; grade 3, disruption and loss of granulosa and theca cells, pyknotic nuclei, and missing oocytes.

\section{Assessment of apoptotic follicles by TUNEL assay}

The remaining paraffin section slides $(n=77)$ were stained using a terminal deoxynucleotidyl transferase-mediated deoxyuridine triphosphate nick-end labeling (TUNEL) assay kit (in situ cell death detection kit; Roche, Mannheim, Germany). In brief, after deparaffinization and rehydration, the sections were rinsed with DPBS and treated with $0.8 \%$ proteinase K (Dako, Glostrup, Denmark) at room temperature for 15 minutes, and then incubated with a TUNEL reaction mixture for 1 hour in a $37^{\circ} \mathrm{C}$ humidified chamber in the dark. After washing, the sections were mounted with Vectashield mounting medium
G1

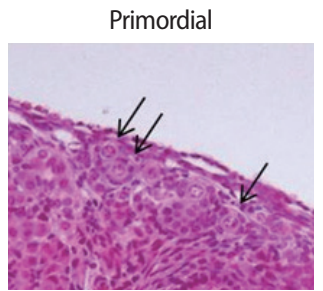

$\mathrm{G} 2$

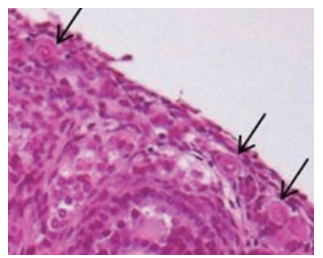

G3

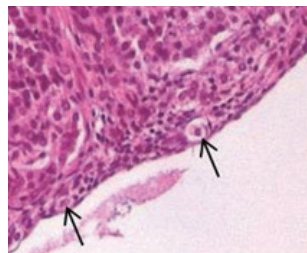

Primary
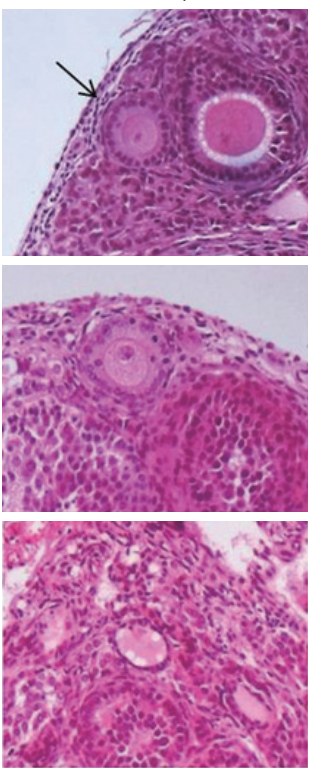

Secondary
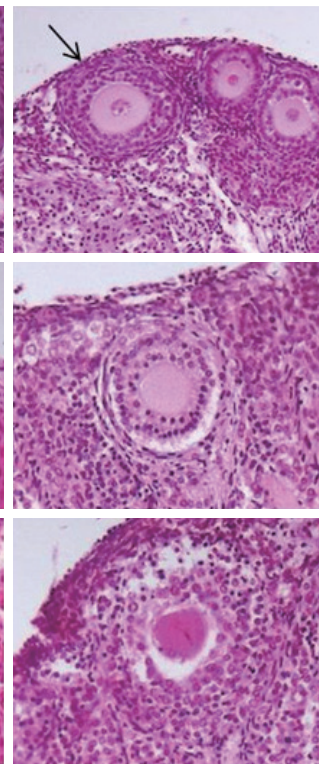

Antral
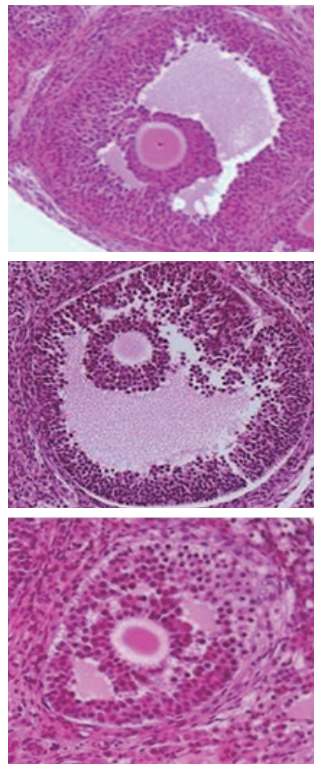

Figure 1. Morphological classification of mouse ovarian follicles stained with hematoxylin and eoxin (original magnification $\times 200$ ). The horizontal axis represents the developmental stage of the follicles. The vertical axis represents the quality of the follicles. G1, good; G2, fair; G3, bad. Each arrow indicates the corresponding developmental stage and quality of the follicle.
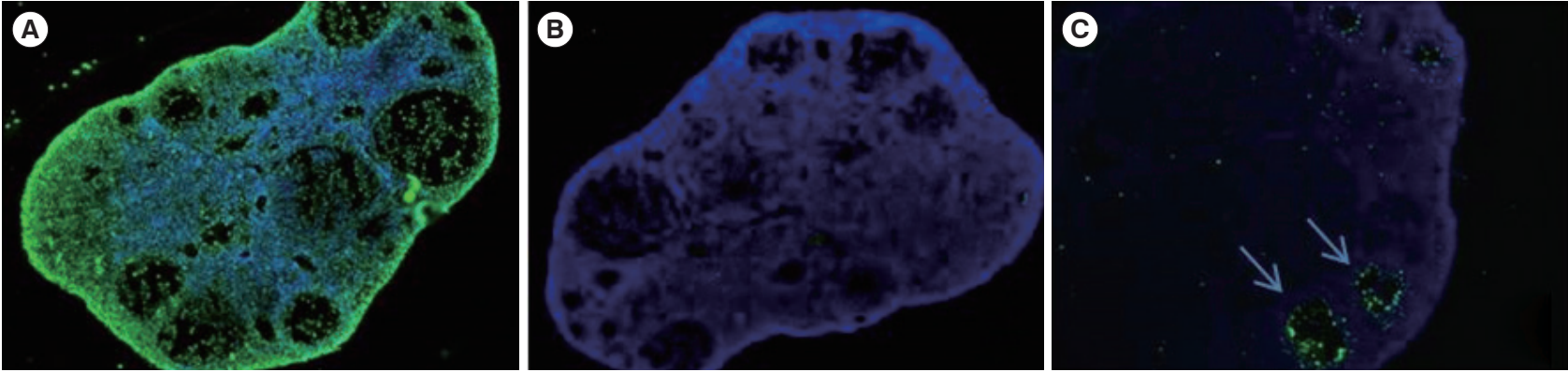

Figure 2. Representative image of fluorescent terminal deoxynucleotidyl transferase-mediated deoxyuridine triphosphate nick-end labeling (TUNEL) staining on vitrified-warmed ovaries (original magnification $\times 100$ ). (A) A positive control. (B) A negative control. (C) TUNEL-positive follicles (arrows). 
with 4,6-diamidino-2-phenylindole (Vector Laboratories, Burlingame, CA, USA) and visualized under an inverted Zeiss AX10 fluorescence microscope (Carl Zeiss, Oberkochen, Germany). Green fluorescence was detected in the follicular cells containing fragmented DNA at an excitation wavelength of 450 to $500 \mathrm{~nm}$ and a detection wavelength of 515 to $565 \mathrm{~nm}$. Blue fluorescence was visualized in the counterstained normal follicular cells at an excitation wavelength of $360 \mathrm{~nm}$ and an emission wavelength of $460 \mathrm{~nm}$. As shown in Figure
2, when green fluorescence was detected in more than $30 \%$ of a single follicle, the follicle was regarded as apoptotic, according to previously published guidelines $[7,9,22]$. Three slides could not be counted due to staining errors.

\section{Statistical analysis}

The proportions of intact (grade 1) follicles overall and according to follicular stage, as well as of apoptotic follicles as a whole in each

Table 2. The proportion of morphologically intact follicles (grade 1) in vitrified-warmed mouse ovaries supplemented with two types of AFP or a combination thereof

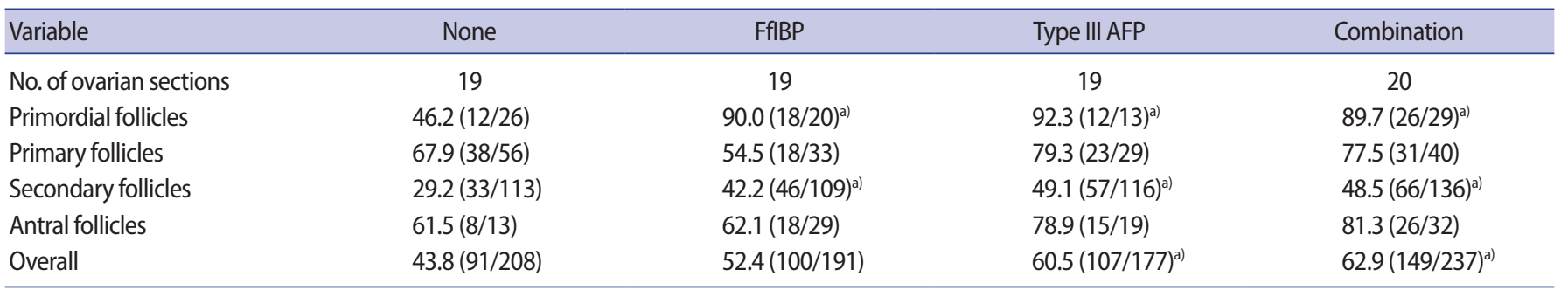

Values are presented as percent (number).

AFP, antifreeze protein; FfBP, Flavobacterium frigoris ice-binding protein.

a) $p<0.05$ when compared with the data in the non-supplemented group.
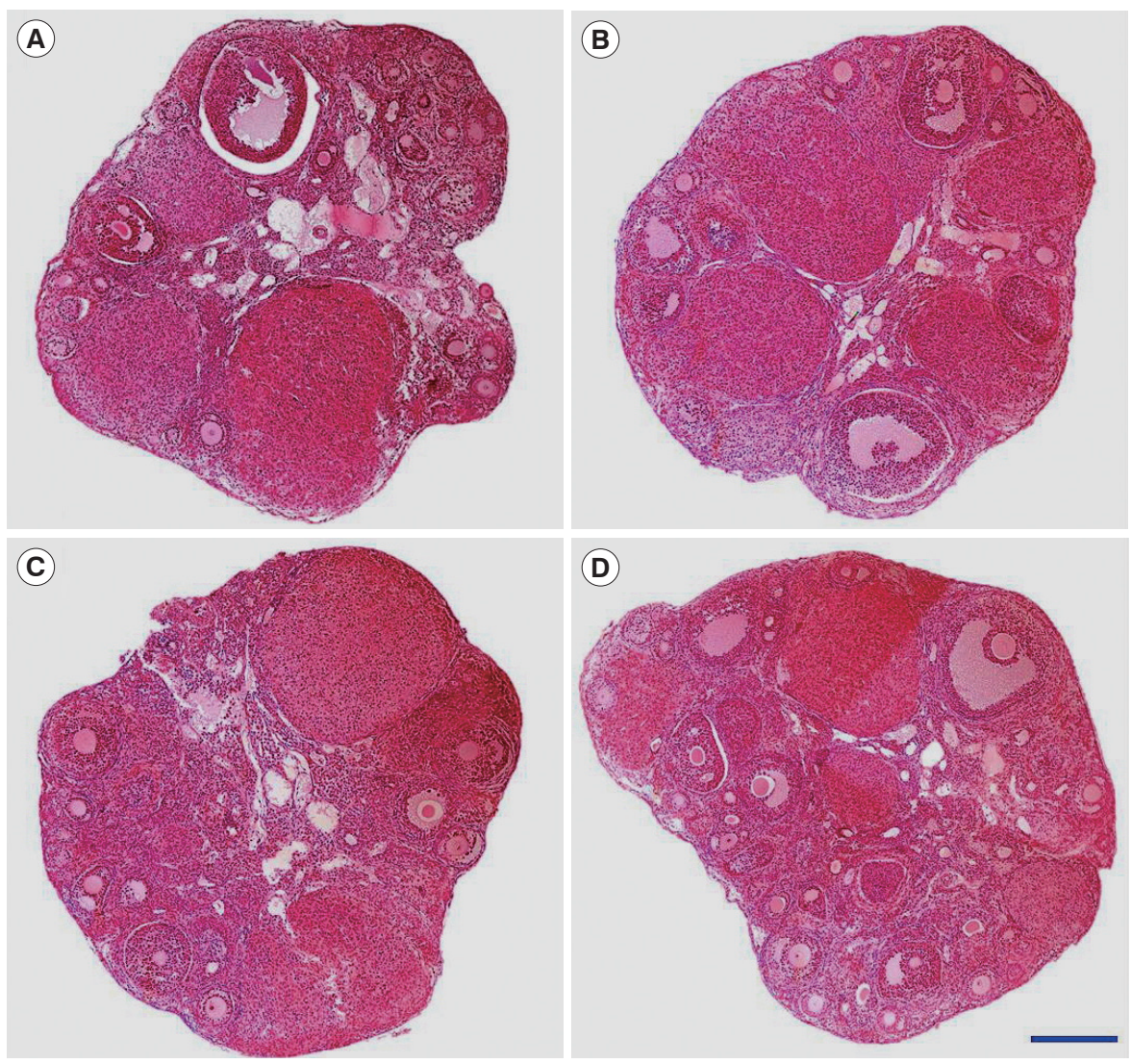

Figure 3. Representative images of hematoxylin and eosin staining from the four groups according to the type of antifreeze protein (AFP) treatment. (A) Vitrified-warmed control. (B) Group treated with $10 \mathrm{mg} / \mathrm{mL}$ of Flavobacterium frigoris ice-binding protein (FflBP). (C) Group treated with $10 \mathrm{mg} / \mathrm{mL}$ of type III AFP. (D) Combination group ( $10 \mathrm{mg} / \mathrm{mL}$ of FfIBP+10 mg/mL of type III AFP). The blue bar indicates $500 \mu \mathrm{m}$. 
Table 3. The proportion of non-apoptotic follicles in vitrified-warmed mouse ovaries supplemented with two types of AFP or the combination thereof

\begin{tabular}{|c|c|c|c|c|}
\hline Variable & None & FflBP & Type III AFP & Combination \\
\hline No. of ovarian sections & 18 & 19 & 18 & 19 \\
\hline No. of total follicles counted & 186 & 234 & 183 & 197 \\
\hline No. of non-apoptotic follicles & 152 & 204 & 162 & 173 \\
\hline Proportion of non-apoptotic follicles ${ }^{\mathrm{a})}(\%)$ & 81.7 & 87.2 & 88.5 & 87.8 \\
\hline
\end{tabular}

AFP, antifreeze protein; FfIBP, Flavobacterium frigoris ice-binding protein.

a)The proportions of non-apoptotic follicles were similar across the four groups.

ovarian section, were calculated and compared using the chi-square test. Data were analyzed using SPSS ver. 18.0 (SPSS Inc., Chicago, IL, USA). The $p$-values $<0.05$ were considered to indicate statistical significance.

\section{Results}

The proportion of intact follicles (grade 1) as a whole was significantly higher in the type III AFP-supplemented group and the combination group, and the proportion of intact primordial or secondary follicles was significantly higher in all three AFP-supplemented groups than in the non-supplemented control group (Table 2). However, the proportions of intact primary and antral follicles were similar between each AFP-supplemented group and the control. Ovarian tissue images from the four groups are provided in Figure 3.

Among the three AFP-supplemented groups, the proportions of intact follicles as a whole and according to follicular stage were all similar.

The proportions of non-apoptotic (TUNEL-negative) follicles as a whole were all similar between each AFP-supplemented group and the control group (Table 3).

\section{Discussion}

In the present study, supplementation of the vitrification and warming solutions with FflBP, type III AFP, or the combination thereof was found to have beneficial effects on the preservation of follicles overall and primordial follicles in particular in vitrified mouse ovaries. The proportions of intact follicles (as a whole or among the primordial follicles) were similar between the FfIBP-supplemented and the type III AFP-supplemented-groups, and the results from both groups were also similar to those obtained from the combination group; thus, the combination of FfIBP and type III AFP appears to be unnecessary for the cryoprotection of mouse ovaries during vitrification.

We initially expected that the combination of FflBP and type III AFP would yield an enhanced outcome in comparison to the use of FFIBP or type III AFP alone due to their complementary synergic effects.
FfIBP and type III AFP differently act on different ice surfaces because each AFP type is distinct in terms of structure and AFP-ice binding area, and such structural diversity leads to different structure-function relationships $[23,24]$. The combination of two AFPs might result in a relatively higher concentration of cryoprotectants, meaning that the synergistic effects may have counterbalanced each other. It is well known that overly high concentrations of cryoprotectants induce considerable osmotic stress and result in follicle damage in cryopreserved mouse ovaries [25].

In the field of fertility preservation, primordial follicles are considered ideal for preservation because they are the most abundant in the ovary and are resistant to the cryopreservation-thawing procedure due to their small size, lack of organelles, and metabolically quiescent stage $[26,27]$. From this point of view, FflBP and type III AFP were equally effective in preserving primordial follicles in vitrified mouse ovaries.

In the present study, the overall morphological follicular integrity was much better in the AFP-supplemented groups; however, the proportions of non-apoptotic follicles were similar to those of the non-supplemented control group. This indicates that the follicles in the vitrified-warmed mouse ovaries may have been damaged, as indicated by apoptotic events, despite being morphologically intact.

In the present study, AFP treatment improved the integrity of primordial and secondary follicles, but not the integrity of primary and antral follicles. The reasons for this are largely unknown, but the relatively small number of primary and antral follicles may have affected the results. When we performed histologic assessments of the ovarian follicles, we examined only one section from each ovary. This avoided duplicated counts from the same follicle, but may have resulted in a smaller number of specific types of follicle in individual ovarian sections. However, we used a relatively large number of mice to reduce such problems.

In the present study, vitrified-warmed mouse ovaries underwent a 30-minute post-warming incubation procedure to stabilize the tissues. During the vitrification and warming, mouse ovarian tissues may be damaged due to ice crystal formation and/or recrystallization. During vitrification, cells or tissues are transformed into a glass- 
like form without the formation of ice crystals, but ice recrystallization can actually occur during the warming process [28]. Post-warming short-term incubation can reduce ice recrystallization during the warming process. In our previous studies, we tested various times of post-warming incubation in vitrified mouse ovaries and found that 30 minutes of post-warming incubation was optimal $[7,18]$. Therefore, we suggest that vitrified-warmed mouse ovaries undergo a 30-minute incubation procedure before transplantation.

In conclusion, to the best of our knowledge, this is the first report to evaluate the cryoprotective effects of FfIBP, type III AFP, and the combination thereof on the vitrification of mouse ovaries. We demonstrated that supplementation with FfIBP, type III AFP, and the combination thereof had comparable effects on follicle integrity enhancement as a whole, as well as in primordial follicles specifically. Further studies are necessary to verify the cryoprotective mechanism of AFPs.

\section{Conflict of interest}

No potential conflict of interest relevant to this article was reported.

\section{References}

1. Byrne J, Kessler LG, Devesa SS. The prevalence of cancer among adults in the United States: 1987. Cancer 1992;69:2154-9.

2. Donnez J, Bassil S. Indications for cryopreservation of ovarian tissue. Hum Reprod Update 1998;4:248-59.

3. Rao GD, Chian RC, Son WS, Gilbert L, Tan SL. Fertility preservation in women undergoing cancer treatment. Lancet 2004;363:182930.

4. Kim SS. Fertility preservation in female cancer patients: current developments and future directions. Fertil Steril 2006;85:1-11.

5. Jeruss JS, Woodruff TK. Preservation of fertility in patients with cancer. N Engl J Med 2009;360:902-11.

6. Faustino LR, Santos RR, Silva CM, Pinto LC, Celestino JJ, Campello CC, et al. Goat and sheep ovarian tissue cryopreservation: effects on the morphology and development of primordial follicles and density of stromal cell. Anim Reprod Sci 2010;122:90-7.

7. Youm HW, Lee JR, Lee J, Jee BC, Suh CS, Kim SH. Optimal vitrification protocol for mouse ovarian tissue cryopreservation: effect of cryoprotective agents and in vitro culture on vitrified-warmed ovarian tissue survival. Hum Reprod 2014;29:720-30.

8. Kagawa N, Silber S, Kuwayama M. Successful vitrification of bovine and human ovarian tissue. Reprod Biomed Online 2009;18: 568-77.

9. Jee BC, Lee JR, Youm H, Suh CS, Kim SH, Moon SY. Effect of sphingosine-1-phosphate supplementation on follicular integrity of vitrified-warmed mouse ovarian grafts. Eur J Obstet Gynecol Reprod Biol 2010;152:176-80.

10. Yeh Y, Feeney RE. Antifreeze proteins: structures and mechanisms of function. Chem Rev 1996;96:601-18.

11. DeVries AL, Wohlschlag DE. Freezing resistance in some Antarctic fishes. Science 1969;163:1073-5.

12. Prathalingam NS, Holt WV, Revell SG, Mirczuk S, Fleck RA, Watson PF. Impact of antifreeze proteins and antifreeze glycoproteins on bovine sperm during freeze-thaw. Theriogenology 2006;66:1894-900.

13. Lee HH, Lee HJ, Kim HJ, Lee JH, Ko Y, Kim SM, et al. Effects of antifreeze proteins on the vitrification of mouse oocytes: comparison of three different antifreeze proteins. Hum Reprod 2015;30: 2110-9.

14. Lee SG, Koh HY, Lee JH, Kang SH, Kim HJ. Cryopreservative effects of the recombinant ice-binding protein from the arctic yeast Leucosporidium sp. on red blood cells. Appl Biochem Biotechnol 2012;167:824-34.

15. Rubinsky B, Arav A, Devries AL. The cryoprotective effect of antifreeze glycopeptides from antarctic fishes. Cryobiology 1992;29: 69-79.

16. Wu Y, Fletcher GL. Efficacy of antifreeze protein types in protecting liposome membrane integrity depends on phospholipid class. Biochim Biophys Acta 2001;1524:11-6.

17. Bagis H, Akkoc T, Tass A, Aktoprakligil D. Cryogenic effect of antifreeze protein on transgenic mouse ovaries and the production of live offspring by orthotopic transplantation of cryopreserved mouse ovaries. Mol Reprod Dev 2008;75:608-13.

18. Borges EN, Silva RC, Futino DO, Rocha-Junior CM, Amorim CA, Bao SN, et al. Cryopreservation of swine ovarian tissue: effect of different cryoprotectants on the structural preservation of preantral follicle oocytes. Cryobiology 2009;59:195-200.

19. Lee J, Kim SK, Youm HW, Kim HJ, Lee JR, Suh CS, et al. Effects of three different types of antifreeze proteins on mouse ovarian tissue cryopreservation and transplantation. PLoS One 2015;10: e0126252.

20. Lundy T, Smith P, O'Connell A, Hudson NL, McNatty KP. Populations of granulosa cells in small follicles of the sheep ovary. J Reprod Fertil 1999;115:251-62.

21. Gandolfi F, Paffoni A, Papasso Brambilla E, Bonetti S, Brevini TA, Ragni G. Efficiency of equilibrium cooling and vitrification procedures for the cryopreservation of ovarian tissue: comparative analysis between human and animal models. Fertil Steril 2006; 85 Suppl 1:1150-6.

22. Lee JR, Youm HW, Lee HJ, Jee BC, Suh CS, Kim SH. Effect of antifreeze protein on mouse ovarian tissue cryopreservation and transplantation. Yonsei Med J 2015;56:778-84. 
23. Chao H, Sonnichsen FD, DeLuca Cl, Sykes BD, Davies PL. Structure-function relationship in the globular type III antifreeze protein: identification of a cluster of surface residues required for binding to ice. Protein Sci 1994;3:1760-9.

24. Jia Z, Davies PL. Antifreeze proteins: an unusual receptor-ligand interaction. Trends Biochem Sci 2002;27:101-6.

25. Zhang JM, Li LX, Liu XL, Yang YX, Wan XP. Sucrose affecting successful transplantation of vitrified-thawed mouse ovarian tissues. J Assist Reprod Genet 2009;26:137-42.
26. Anderson RA, Wallace WH, Baird DT. Ovarian cryopreservation for fertility preservation: indications and outcomes. Reproduction 2008;136:681-9.

27. Gosden RG, Mullan J, Picton HM, Yin H, Tan SL. Current perspective on primordial follicle cryopreservation and culture for reproductive medicine. Hum Reprod Update 2002;8:105-10.

28. Seki S, Mazur P. Effect of warming rate on the survival of vitrified mouse oocytes and on the recrystallization of intracellular ice. Biol Reprod 2008;79:727-37. 